
قياس الكفاءة التسويقية لمحصول التفاح في شعبية* الجبل الأخضر في ليبيا

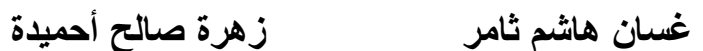

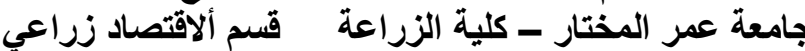
قَمم الاقتصاد الزراعي

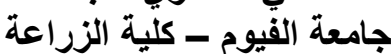

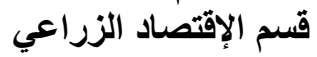

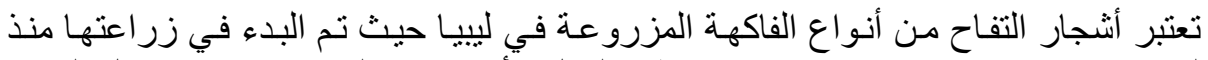

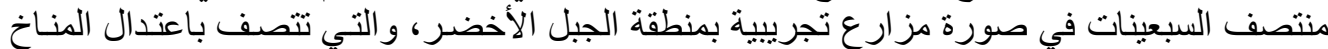

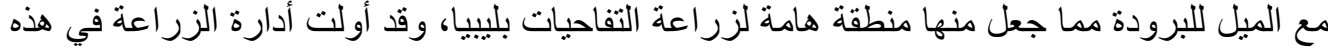

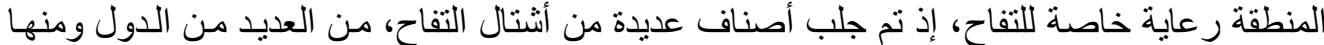

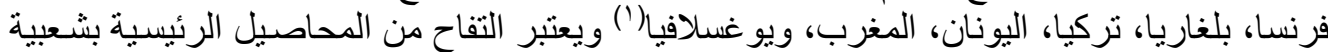

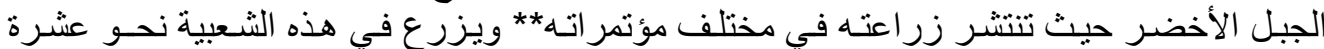

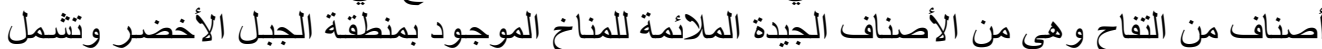

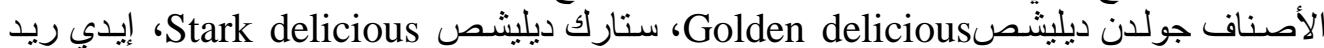

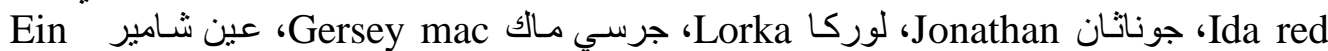

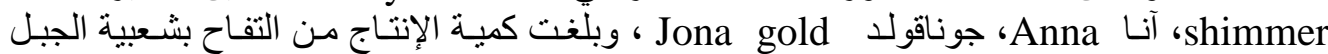

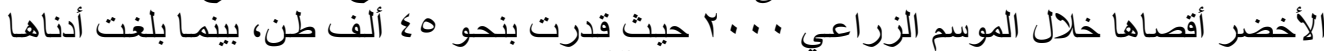

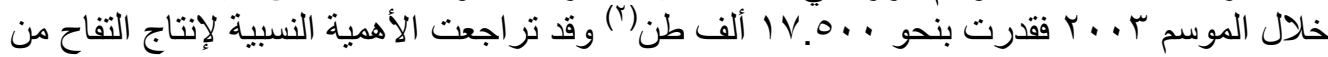

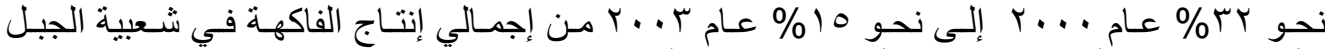

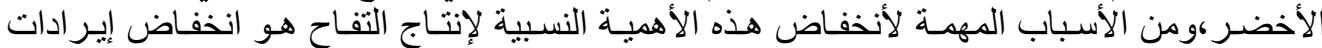

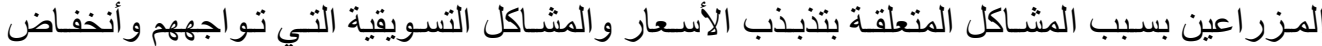
الكفاءة التسويقية للتفاح المنتج و المسوق محلياً.

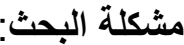

بمكن تحديد مشكلة البحث في انخفاض إير ادات المزارعين لمحصول التفاح في شعبية الجبل

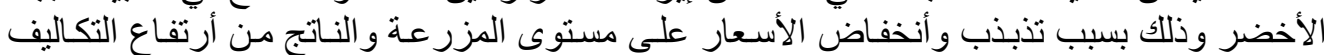

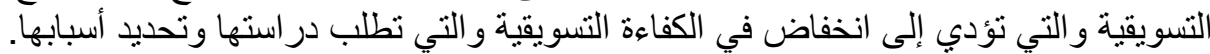

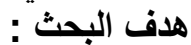

1- يهدف البحث إلى تقدير و قياس الكفاءة التسويقية لمحصول التفاح المنتج محلياً في شعبية الجبل

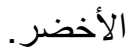

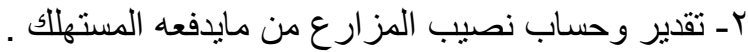
مصادر البيانات والطريقة البحثية:

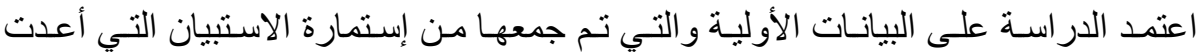

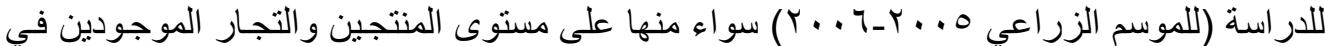

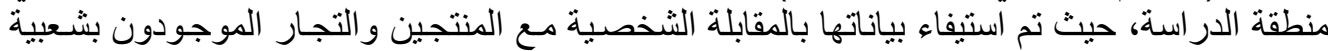

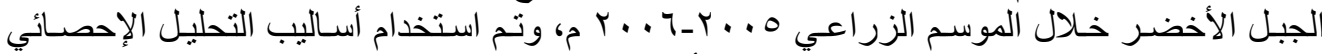

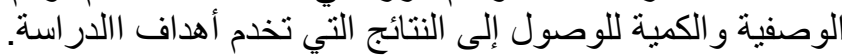
وصف مجتمع الاراسة وأختيار العينة النئة

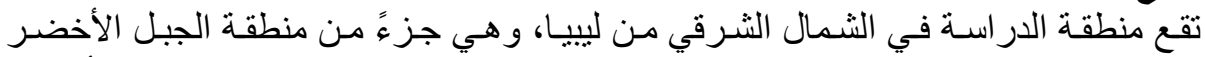

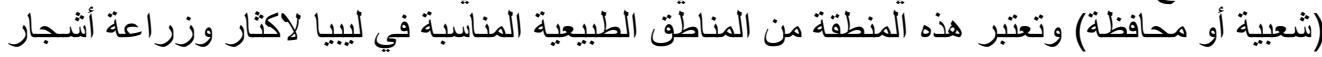
( (1) صلاح منصور بن عبسي، دراسة لررض التقر ح على أشجار الثناح في منطقة الجبل الأخضر، رسالة ماجستير، قسم

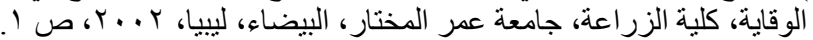

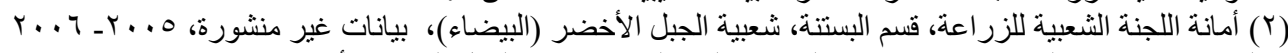

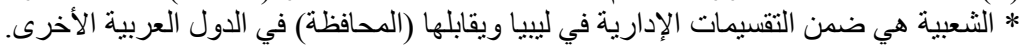
ا ** المؤتمر هو من الناحية الإدارية يعادل المركز في مصر و القضاء في العياء في العراق.

Fayoum J. Agric. Res. \& Dev., Vol.23, No.1, B. January, 2009 


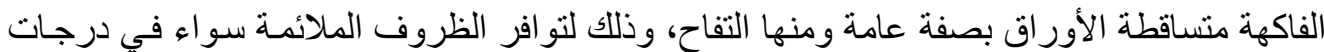

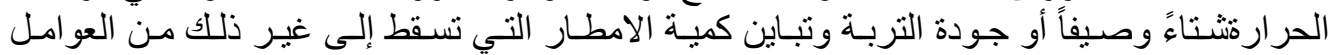

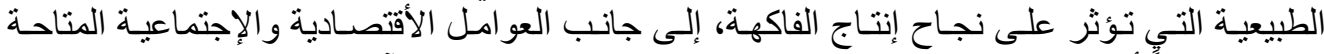

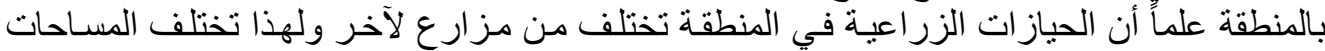
المزرو عة بالتفاح من مزر اليطة إلى أخرى.

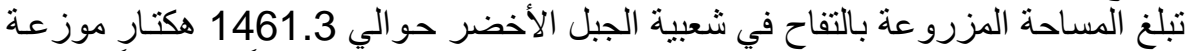

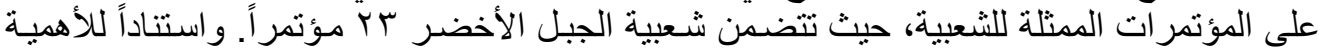

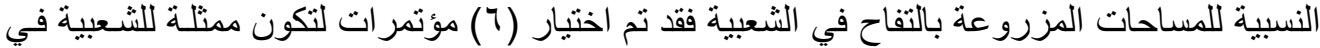

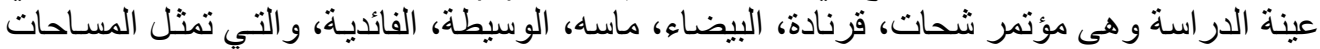

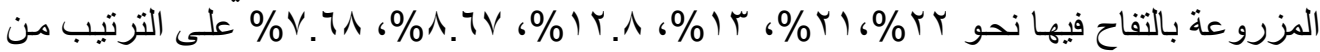

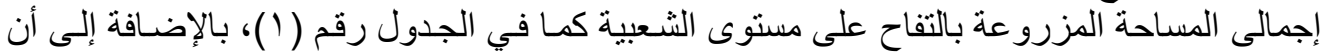

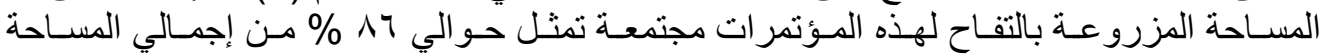

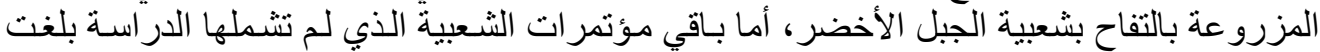

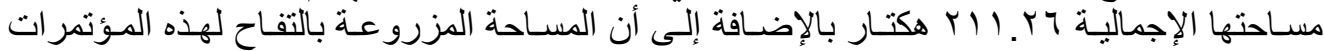

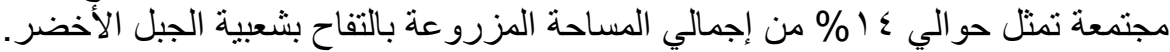

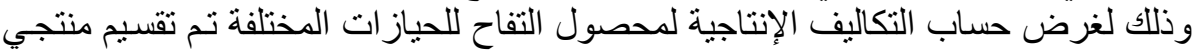
التفاح في الشعبية إلى أربعة فئات حيازية من خلال تطبيق أسلوب المعاينة العشوائية الطبقية المنتظمـة و وهذه الفئات هي:-

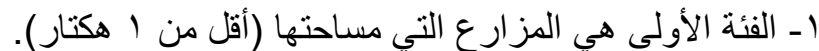

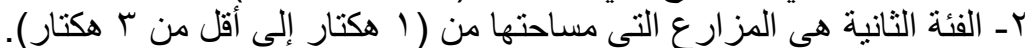

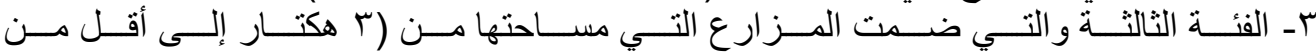
7 الركتار ). ـ - الفئة الر ابعة من ( آ هكتار فأكثر).

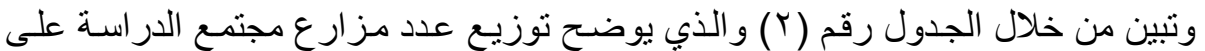

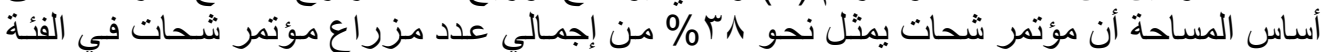

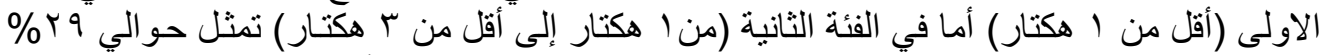

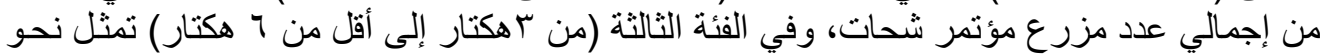

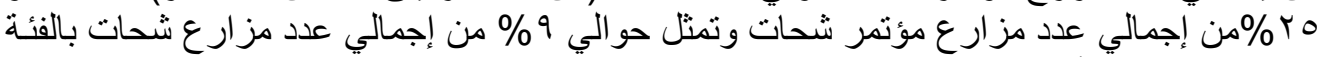

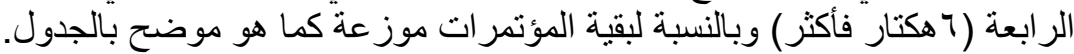

جدول رقم ( )): الأهمية النسبية للمسـاحة المزروعة بالتفاح في المؤتمرات التابعه لثسعبية الجبل

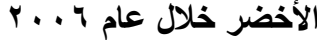

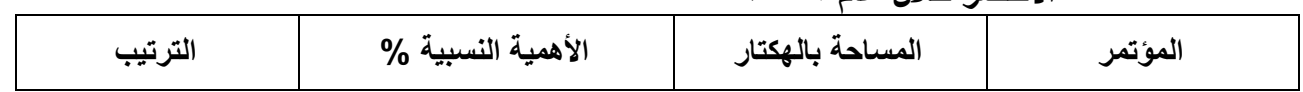

Fayoum J. Agric. Res. \& Dev., Vol.23, No.1, B. January, 2009 
$\varepsilon V$

\begin{tabular}{|c|c|c|c|}
\hline 1 & rr.rq & TYO.AT & شحات \\
\hline$r$ & YI. I & 5.7 .9$. & قرنـادة \\
\hline$r$ & $1 Y . \cdot T$ & $19 \cdot .19$ & البيضاء \\
\hline$\varepsilon$ & IY.AN & $1 \wedge \wedge .10$ & ماسه \\
\hline 0 & $1.7 \mathrm{~V}$ & IYY.VY & الوسيطة \\
\hline 7 & V.71 & IIY.YY & الفائدية \\
\hline$v$ & r.0 & $01 . \vee \wedge$ & اققنطة \\
\hline$\Lambda$ & Y.Yq & rr.01 & قصر ليبيا \\
\hline 9 & $1.7 \varepsilon$ & $Y \leqslant . \cdot Y$ & اسلنطة \\
\hline 1. & $1 . \varepsilon$ & $r \cdot .0$ & الجهاد \\
\hline 11 & 1.41 & 19.15 & عمر المختار \\
\hline Ir & $1 . Y Y$ & 18.87 & بالحديد \\
\hline ir & $1 . \cdot \varepsilon$ & 10.10 & وردامه \\
\hline $1 \leqslant$ & $\cdot \Lambda$. & $11 . v \varepsilon$ & سيدي عبد الواحد \\
\hline 10 &. $.7 \mathrm{~V}$ & $9.8 V$ & "مراوه \\
\hline 17 & .10 & Y.YT & جردس \\
\hline IV &. $.1 \pi$ & $1 . \wedge 0$ & زاوية العرقوب \\
\hline 11 &. .14 & $1 . V Y$ & الحمامةة \\
\hline 19 & $\because .9$ & $1 . r \Lambda$ & قتلدولة \\
\hline$r \cdot$ & $\because \cdot r$ &. .50 & سوسة \\
\hline YI & $\because \cdot Y$ &..$Y q$ & الحنية \\
\hline & $1 \cdots$ & $1 \leqslant 7 \% .0 V$ & الإجمالي \\
\hline
\end{tabular}

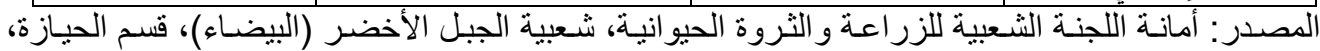

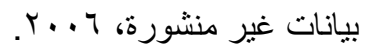

جدول رقم (Y) يبين عدد مزارع مجتمع الاراسة موزعة على على أساس المساحة

\begin{tabular}{|c|c|c|c|c|c|c|c|c|c|c|c|c|}
\hline \multicolumn{2}{|c|}{ الفائدية } & \multicolumn{2}{|c|}{ الوسيطة } & \multicolumn{2}{|c|}{ ماسسه } & \multicolumn{2}{|c|}{ قُرناده } & \multicolumn{2}{|c|}{ البيضاء } & \multicolumn{2}{|c|}{ شحات } & \multirow[t]{2}{*}{ المؤتمر } \\
\hline$\%$ & المزارع عد & $\%$ & | علدادع & $\%$ & المزارع & $\%$ & المزارع عد & $\%$ & المزارع & $\%$ & المزارع & \\
\hline 01 & YT & ir & v & $Y \varepsilon$ & 17 & 19 & 19 & $\leqslant r$ & $\leq 4$ & rs & $\leqslant \wedge$ & أقل من 1 هكتار \\
\hline 4 & 17 & 17 & 9 & $\leqslant r$ & rq & r. & r. & rq & r & rq & 4 & من 1 إلى أقل من r \\
\hline ir & 7 & $\leqslant V$ & $r V$ & $\mu_{1}$ & r) & \& & « & rT & $r \varepsilon$ & ro & r & 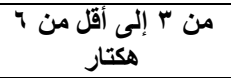 \\
\hline - & - & ro & 10 & r & 1 & 7 & 7 & 0 & 0 & 9 & 11 & من \ هكتار فأكثر \\
\hline & $\leqslant 0$ & & $0 \wedge$ & & IV & & 99 & & 1.7 & & $1 \% 9$ & المجموع \\
\hline
\end{tabular}

المصدر: أمانة اللجنة الشعبيةة للزراعة والثروة الحيوانية، شعبية الجبل الأخضر (البيضساء)، قسم الحيازة، بيانـات غير

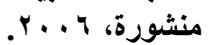

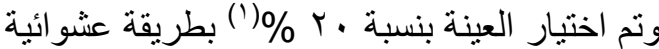

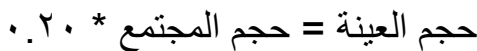

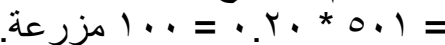

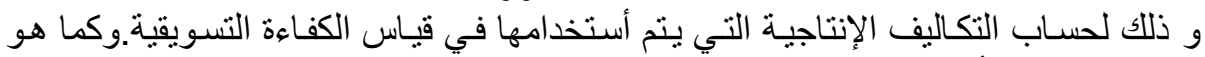

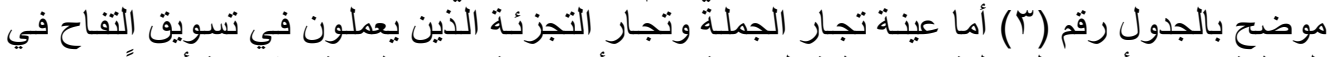
المنطقة وفي الأحياء المختلفة من منطقة الدر اسة فقد تم أخذ عينة منهم بطريقة عشو ائية أيضاً.

$$
\text { جدول رقم (r) توزيع عدد المزارع حسب المساحة لكل مؤتمر شعبي }
$$

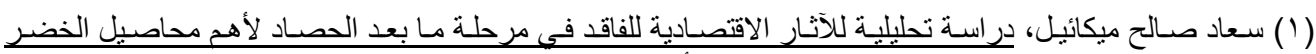

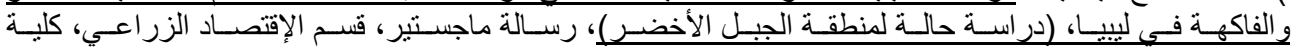

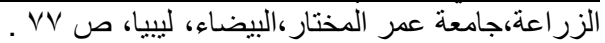

Fayoum J. Agric. Res. \& Dev., Vol.23, No.1, B. January, 2009 
$\varepsilon \wedge$

\begin{tabular}{|c|c|c|c|c|c|c|c|}
\hline العدد الكلي لمفردات & الفائدية & الوسيطة & ماسه & قرنادة & البيضاء & شحات & المنطقة \\
\hline rr & 0 & 1 & $r$ & $\varepsilon$ & 9 & 1. & أقل من 1 هكتار \\
\hline rq & $r$ & $r$ & $\bullet$ & 7 & 7 & V & من I إلى أقل من r هكتار \\
\hline r & 1 & 7 & $\varepsilon$ & 9 & 0 & 7 & من \ إلى أقل من \ هكتار \\
\hline$\wedge$ & - & $r$ & 1 & 1 & 1 & r & من I هكتار فأكثر \\
\hline $1 \cdots$ & 9 & ir & Ir & $r$. & Y & ro & المجمـــــوع \\
\hline
\end{tabular}

المصدر: جمعت وحسبت من الجدول رقم (r)

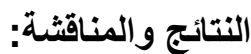

قياس الكفاءة التسويقية لمحصول التفاح في شعبية الجبل الأخضر:

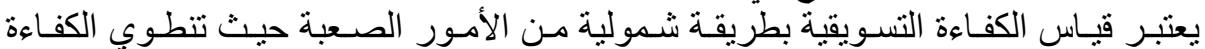

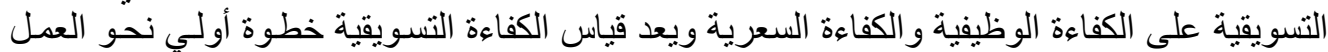

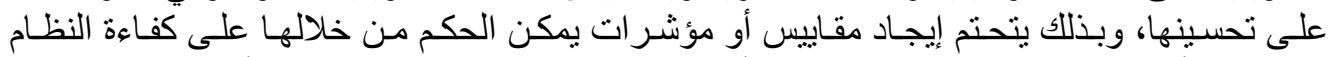

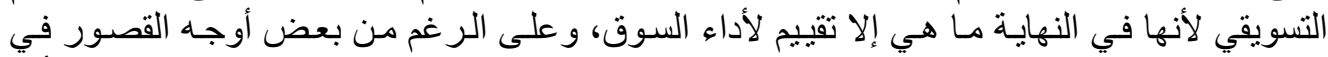

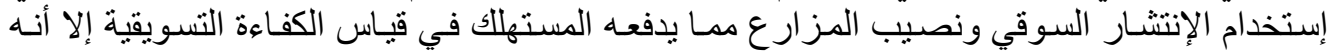

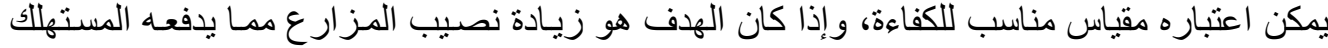

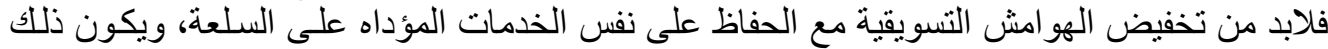

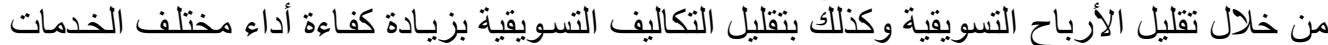

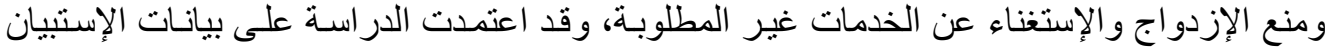

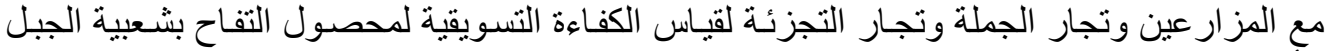

الأخضر ، وتقدير نصيب المز ارع من سعر المر المستهلك وكذللك التكاليف التسويقية من سعر المستهلك.

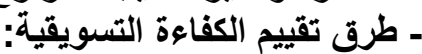

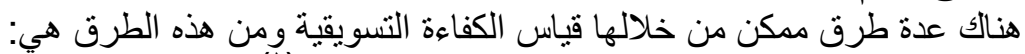

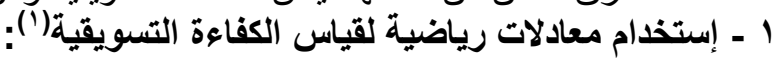

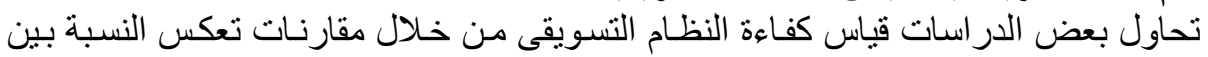
جملة التكاليف التسويقية، و التكاليف الكلية (الإنتاجية والتسويفية التية) للمحصول المسوق باستخدام المقياس التكاليف التسويقية

$$
\text { الكفاءة التسويقية = . . 1- }
$$

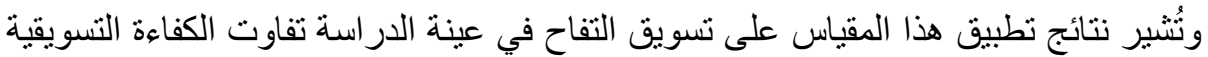

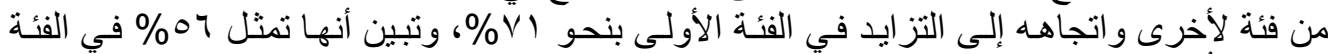

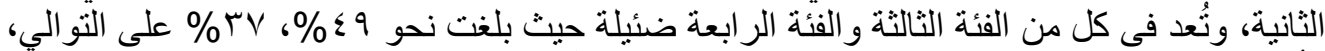

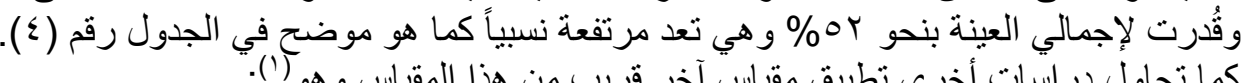

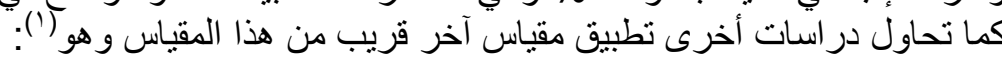

$$
\text { الكفاءة التسويقية = . . 1 الهوامش التسويقية + التكاليف الإنتاجية التسويقية }
$$

(') صبحى محمد اسماعيل، الكفاءة التسويقية لبعض الزروع الخضرية الهامة في جمهورية مصر العربية، رسالة دكتوراه،

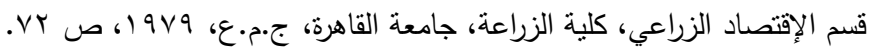

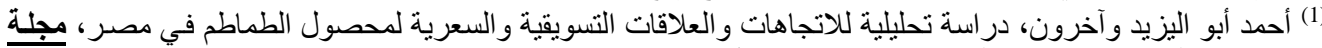

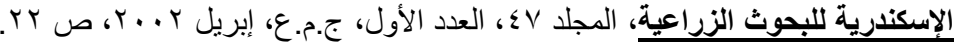

Fayoum J. Agric. Res. \& Dev., Vol.23, No.1, B. January, 2009 


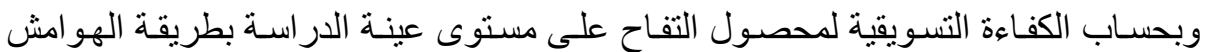

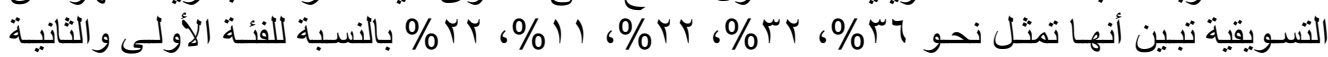

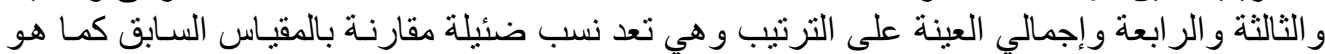

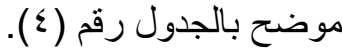
وبتطبيق المعادلة الرياضية لحساب الكفاءة التسويقية تبين أن :

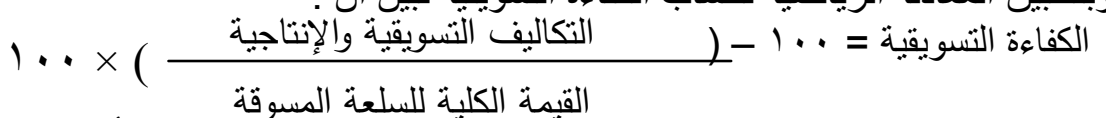

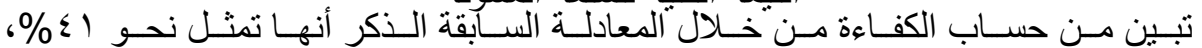

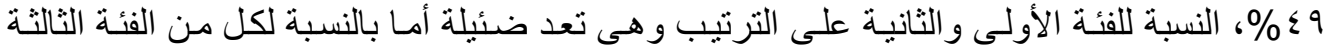

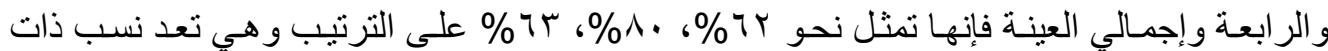

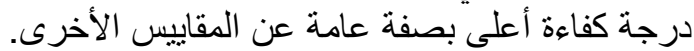

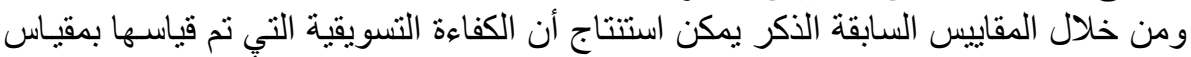

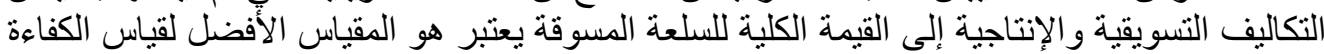

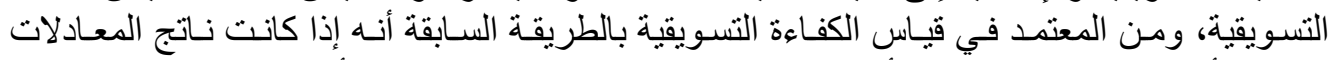

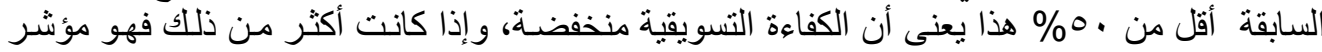
أريفاع كفاءة تسويق السلعة المدروسة.

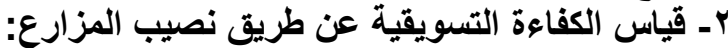
ومن الطرق المستخدمة في قياس الكفاءة التسويقية هو فئية قياس الكفاءة التسويقية عن طريق نصيب

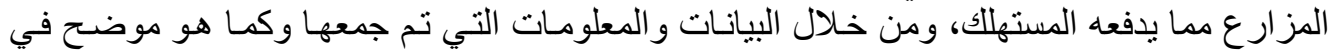

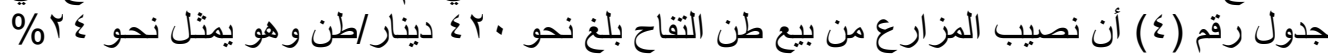

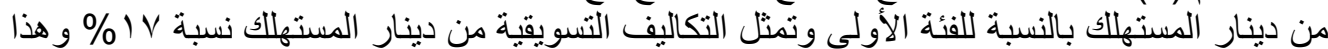

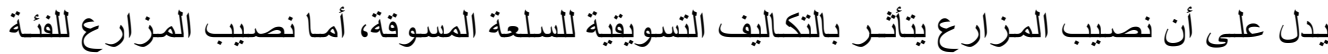

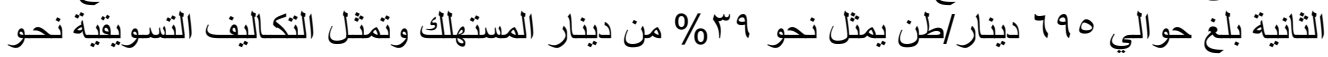

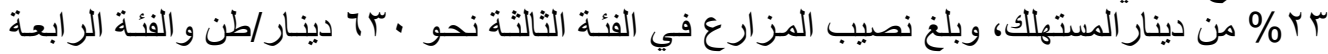

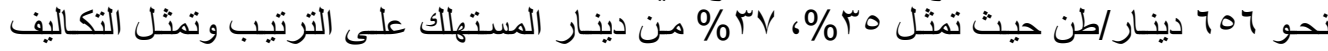

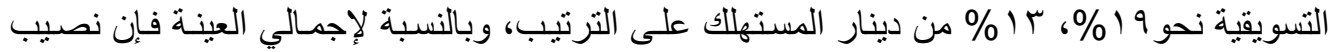

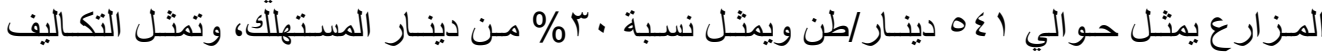

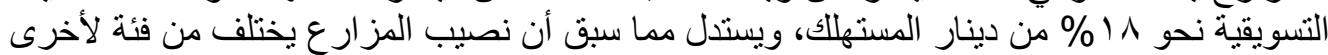

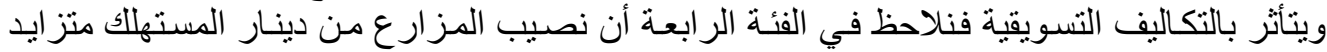

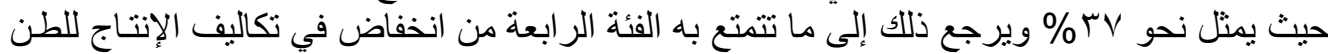

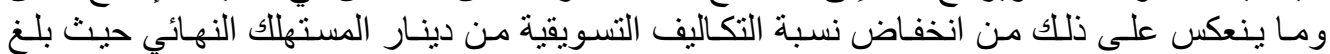
\%

rـ قياس كفاءة اداء الوظائف التسويقية لمحصول التفاح في شعبية الجبل الأخضر بأسلوب الدرجات:

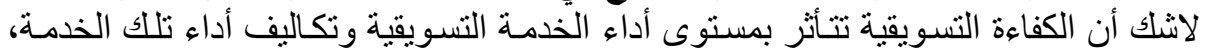

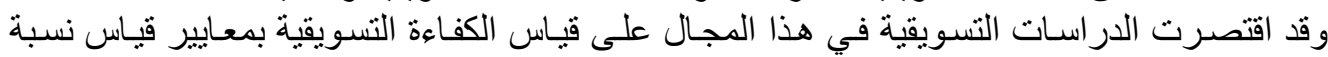

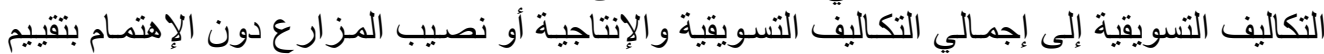

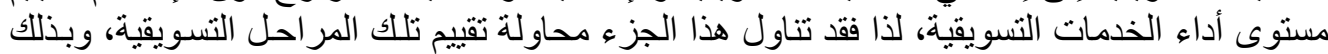

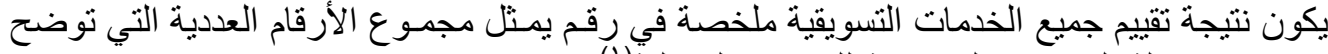

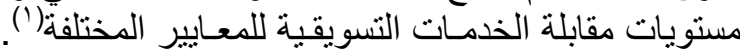

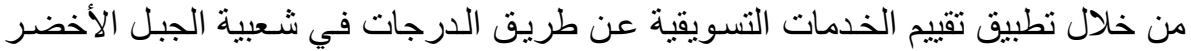

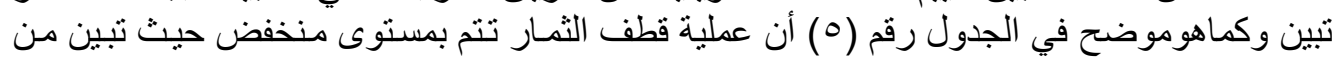

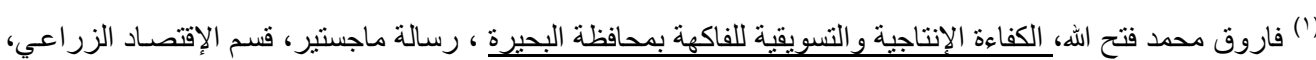

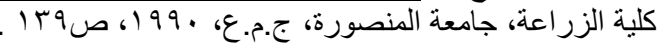

Fayoum J. Agric. Res. \& Dev., Vol.23, No.1, B. January, 2009 
๑.

نتيجــة الإسـتبيان أن . 1\% مـن أفر اد العينـة لا بسـتخدمون المقصـات و المتطلبـات الأخـرى لعمليـة

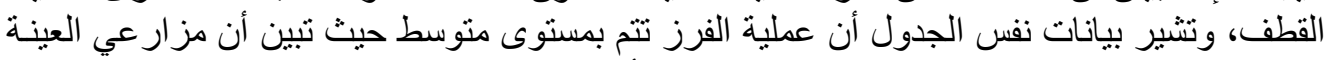

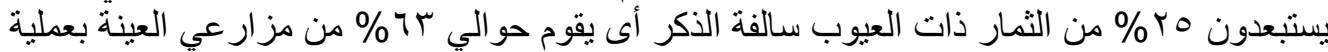

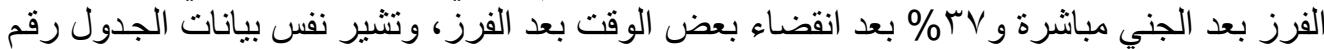

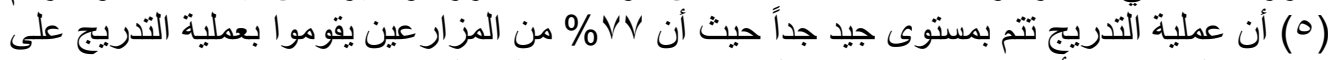

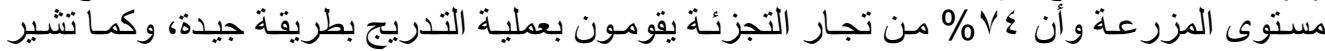

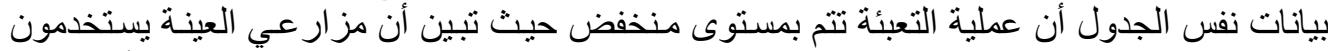

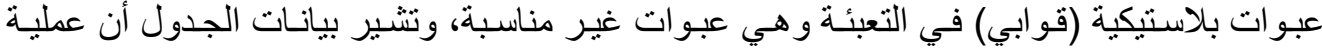

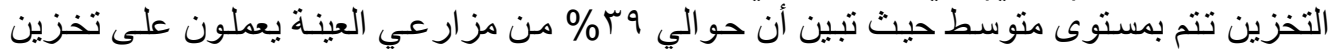

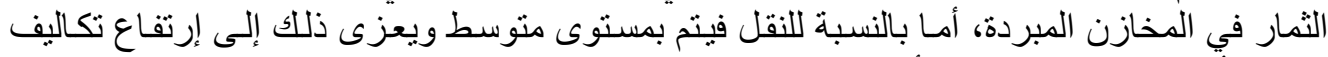

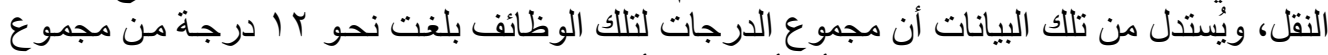

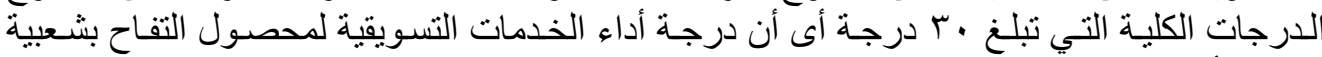

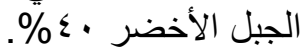
جدول رقم (؛ ): حسـاب الكفـاعة التسـويقية بالمقـاييس التـي تـربط بـين التكـاليف الإتتاجيـة والتسـويقية وقيمـة السـلعة

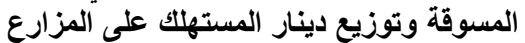

\begin{tabular}{|c|c|c|c|c|c|c|c|c|c|c|c|}
\hline 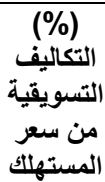 & المن منيب المسترع & التسويقية الكفاعة & الكفاعة التسوية & التسفويقية ألتمة & التسويقية الإلتيفة & التسكاليفية & الإلتهويقي & المستهن: للطن & اللطزر السعي & تلالإنتاج & الفئات \\
\hline IV.. & $r \varepsilon$. & $\& 1$. & rq. & VI.. & 907.8 & 5.7 .70 & $114 . .11$ & $I V A$. & 219.19 & $V \varepsilon q . V V$ & القئة الأولى \\
\hline rr.. & rq. & \&9. & MY.. & 09. & 917.01 & $\varepsilon \cdot T . \leqslant 1$ & $1 . \wedge \varepsilon . \wedge 1$ & IVA. & 790.19 & $01 \% .1 \mathrm{~V}$ & الفئة الثانية \\
\hline $19 .$. & ro.. & TY. & YY.. & $\leqslant 9$. & $7 \vee \leqslant . \wedge 7$ & $r \leqslant 7.0$ & $110 . .1 \varepsilon$ & $I \vee \wedge$. & 749.79 & r૫A.rq & الفئة الثالثة \\
\hline $1 \%$. & $r v_{.} \cdot$ & $\Lambda \cdot$. & 11.0 & rV.. & r4.1\% & YYV.EI & IIr.Vo & $I \vee \wedge$. & 707.10 & IFY.VY & الفئة الر ابعة \\
\hline 11. & r... & 7. & rr. & Or. & $70 \leqslant .10$ & س. & Irrq. & IVA. & $0 \varepsilon . . \vee q$ & & \\
\hline
\end{tabular}

$$
\begin{aligned}
& \text { المصدر: جمعت وحسبت من استثارة الاستبيان }
\end{aligned}
$$

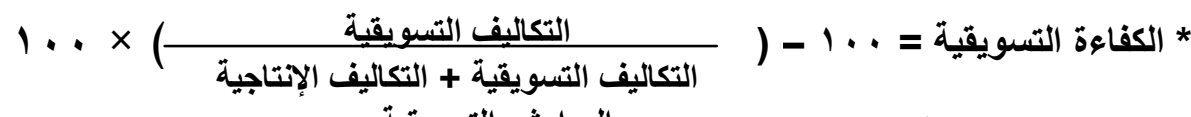

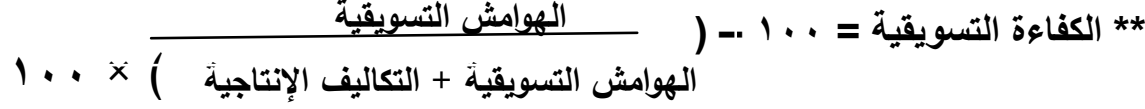

$$
\begin{aligned}
& \text { التكالبف التسويقبة والإنتاجية } \\
& \text { **** الكفاعة التسويقية = . . 1 } \\
& \text { الكفاعة التسويقية بالارجات = }
\end{aligned}
$$

\begin{tabular}{|c|c|c|c|c|}
\hline الدرجات النهائية & الارجة* & المستوى & درجة مطابقة أجراء العملية & الخدمة التسويقية \\
\hline 0 & 1 & منخفض & اقل من oro\% & القطف (الجمع) \\
\hline 0 & r & متوسط & $\%$ ro & الفرز \\
\hline 0 & $\varepsilon$ & جبد جداً & $\% \vee 0$ & التدريج \\
\hline 0 & 1 & منخفض & اقل من OY\%\% & التعبئة \\
\hline
\end{tabular}

جدول رقم (0) تقييم الخدمات التسويقية لمحصول التفاح في شعبية الجبل الاخضر بالدرجات

Fayoum J. Agric. Res. \& Dev., Vol.23, No.1, B. January, 2009 
01

\begin{tabular}{|c|c|c|c|c|}
\hline 0 & $r$ & متوسط & $\%$ Yro & التخزين \\
\hline 0 & $r$ & متوسط & $\%$ Yro & النقل \\
\hline$\mu$ & $1 Y$ & & & الإجمالي \\
\hline
\end{tabular}

المصدر: حسبت من بيانات استمارة الاستبيان.

* أوزان نسبية تم تقدير ها بمعرفة الباحث.

الملخص والتوصيات

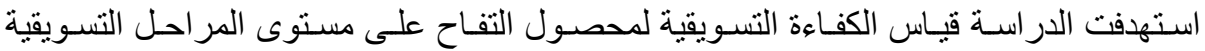

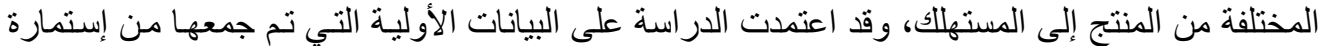

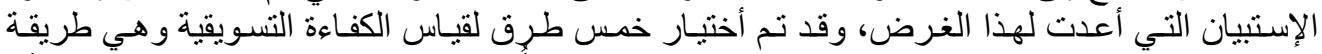

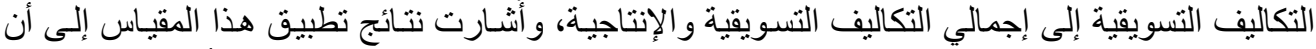

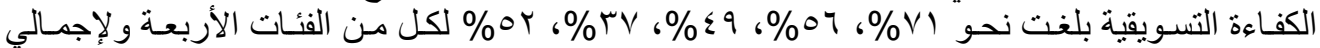

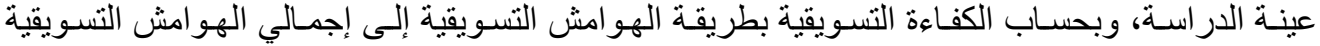

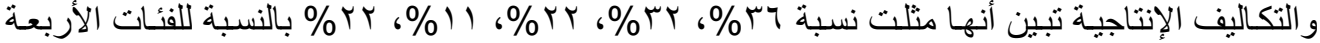

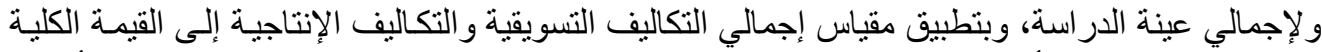

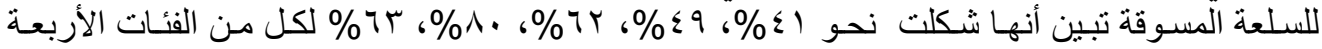

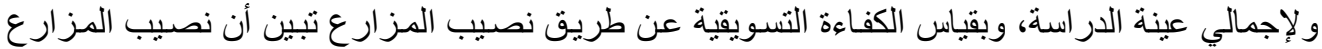

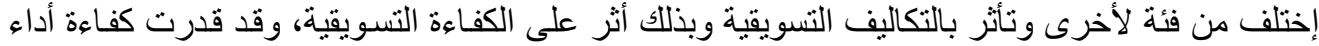

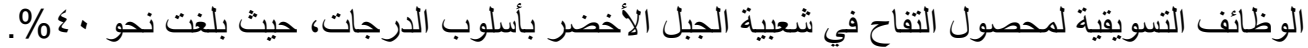

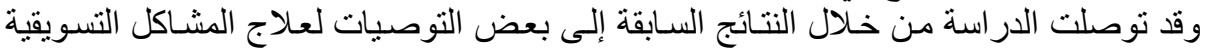

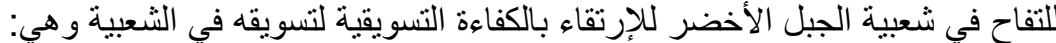

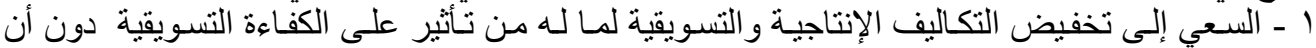

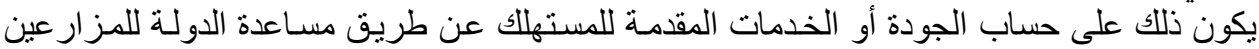

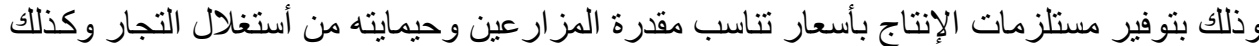

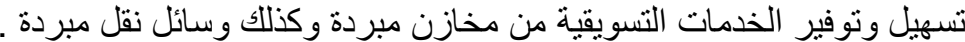

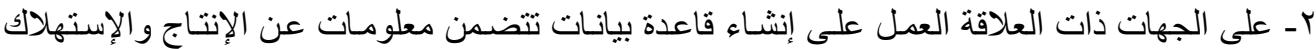

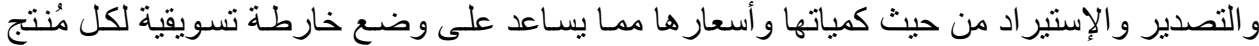

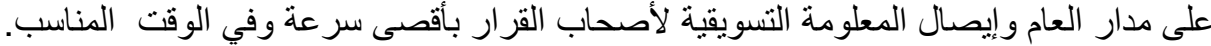

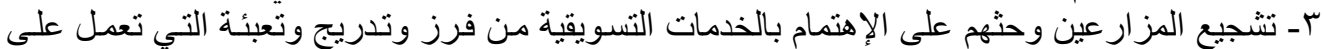

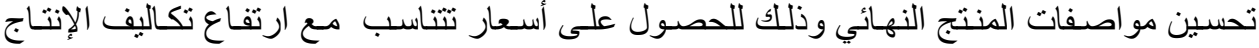

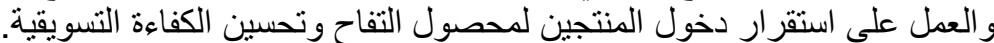

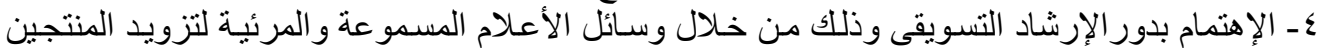

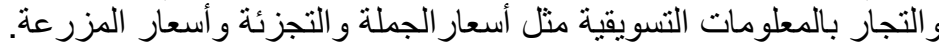

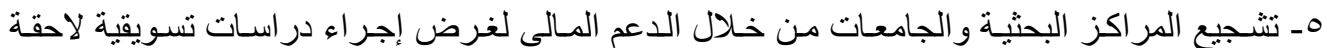

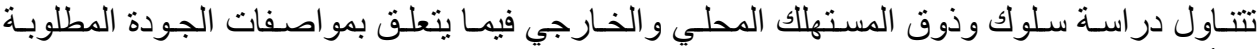

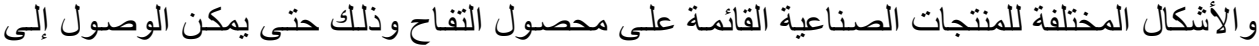
صناعة تصديرية أو معنى أخر الإنتاج من أجل التصائة التصدير.

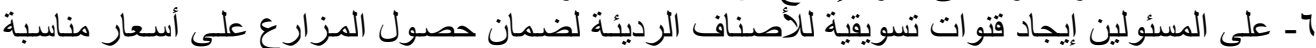
لإنتاجه بكافة درجاته المختلفة.

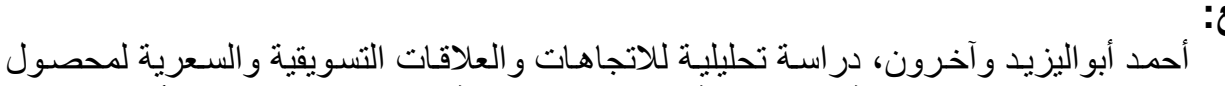

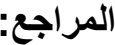

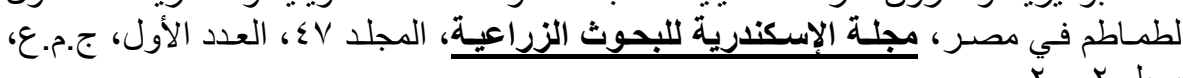

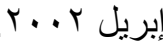

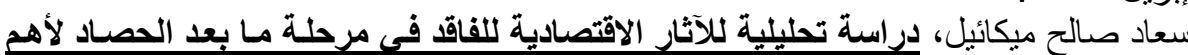

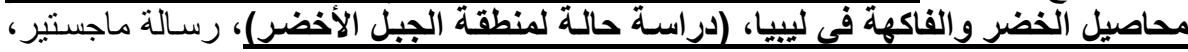
قسم الإقتصاد الزراعي، كلية الزر اعة، جامعة عمر المختار ، ليبيا.

Fayoum J. Agric. Res. \& Dev., Vol.23, No.1, B. January, 2009 


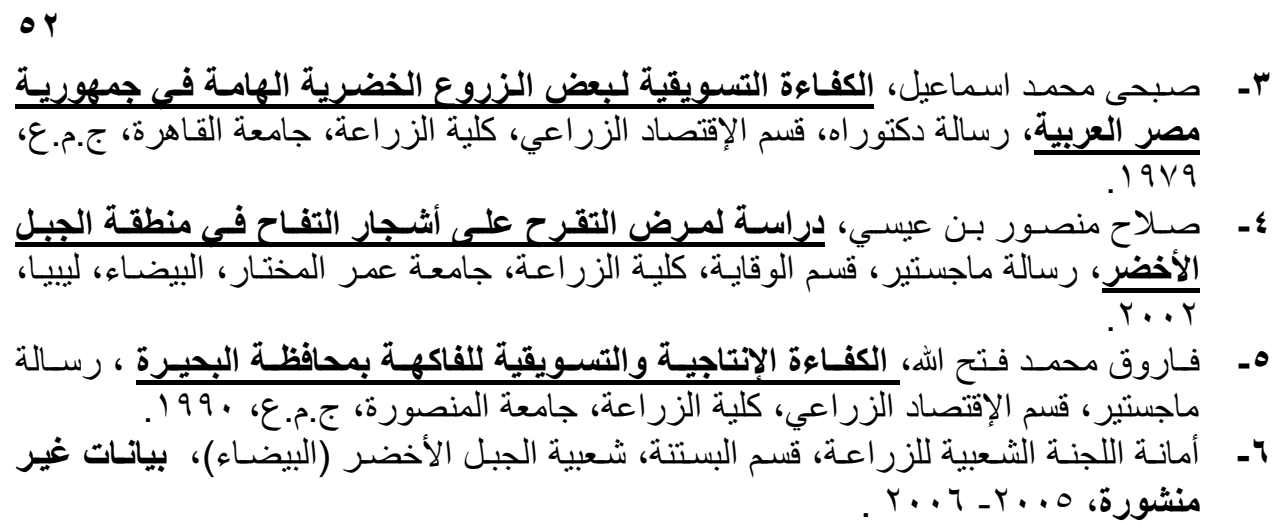

\title{
MEASUREMENT OF MARKTING FFICIENCY OF THE APPLE CROP IN ALJABAL ALAKHDAR MUNICIPALITY
}

\begin{abstract}
The study aimed to measure the marketing efficiency of the apple harvest at various stages of marketing the product to the consumer, The study has adopted on the preliminary data had been collected from the survey form prepared for this purpose, the results of the study has indicated the marketing efficiency of the apple crop in the study sample was collected in five ways. The first one of measuring the marketing efficiency is marketing cost to the total marketing and production cost, and the results indicated by using this measurement that the marketing efficiency amounted to about $71 \%, 56 \%, 49 \%, 37 \%, 52 \%$ for each of the four categories and the total study sample and by using the second method of measuring the marketing efficiency which is the marketing margins to the total marketing margins and production cost, the results were found to be approximately of $36 \%, 32 \%, 22 \%, 11 \%, 22 \%$ for the four categories and for the total sample of the study.

And by application the measurement of the total cost of marketing and production cost to the total coverall value of the marketed product commodity were found to be accounted for about $41 \%, 49 \%, 62 \%, 80 \%, 63 \%$ for each of the four categories and for the total sample of the study. The measurement of efficiency based on the market farmer share, the study showed that the share of the farmers varied from one category to another, and it was affected by marketing cost, and that had an impact on the marketing, efficiency. The marketing functions performing of the apple harvest in the popularity of the AL JABAL AL AKHDAR based on the grading manner was amounted to some of $40 \%$.
\end{abstract}

Fayoum J. Agric. Res. \& Dev., Vol.23, No.1, B. January, 2009 